\title{
DISTRIBUIÇÃO DE POROS POR TAMANHO E SUA RELAÇÃO COM OS PARÂMETROS DE AJUSTES DA CURVA DE RETENÇÃO DE ÁGUA NO SOLO
}

\author{
Pedro Luan Ferreira da Silva ${ }^{1}$, Flávio Pereira de Oliveira ${ }^{2}$, André Julio do Amaral $^{3}$, Walter Esfrain \\ Pereira $^{4}$, Adriana Ferreira Martins ${ }^{5}$, Danillo Dutra Tavares ${ }^{6}$
}

\section{RESUMO}

A distribuição de poros por tamanho exerce influência sobre a dinâmica de gases e solutos do solo, além de atuar sobre o desenvolvimento do sistema radicular das plantas. Contudo, verifica-se que em solos arenosos a distribuição irregular das partículas de areia pode modificar a distribuição de poros e o seu comportamento físico-hídrico. Nesse sentido, o objetivo deste trabalho foi avaliar a relação entre a distribuição de poros no solo por tamanho e os parâmetros de ajuste da curva de retenção de água no solo $(\alpha, n)$ em um Planossolo Háplico sob manejo de produção conservacionista no Agreste da Paraíba. Amostras de solo com estrutura indeformada foram coletadas no solo sob sistema de integração lavoura-pecuária-floresta nas camadas de 0,00-0,10 e 0,10-0,20 m, no município de Alagoinha, estado da Paraíba. Realizou-se a análise granulométrica com o fracionamento da areia em classes de tamanho. Além disso, submeteu-se as amostras aos potenciais $-6,-10,-33,-100,-300,-500,-1000$, e -1500 kPa, para determinação da curva característica de retenção de água no solo e distribuição de poros por tamanho. O Planossolo apresentou predomínio da fração areia nas frações média e fina. A distribuição de poros mostrou-se heterogênea em função do teor de areia. Conclui-se que a distribuição de poros por tamanho apresentou correlação positiva e significativa com os parâmetros de ajustes da curva característica de retenção de água no solo (n) e ( $\alpha$ ).

Palavras:-chave:Porosidade, curva de retenção, Planossolo, ILPF.

\section{PORE DISTRIBUTION BY SIZE AND ITS RELATIONSHIP WITH THE ADJUSTMENT PARAMETERS OF THE WATER RETENTION CURVE IN THE SOIL}

\begin{abstract}
The pore distribution by size influences the gases dynamics and the soil solutes, besides acting on the development of the root system of plants. However, it verifies that in sandy soils the irregular distribution of sand particles can modify the pore distribution and their hydro-physical functioning. Considering this, the objective of this work was to analyze the relationship between the pore distribution in the soil by size and the adjustment parameters of the water retention curve in the soil $(\alpha, n)$ in a Planosols Haplic under management
\end{abstract}

\footnotetext{
${ }^{1}$ Engenheiro Agrônomo, mestrando em Agronomia, Universidade Estadual de Maringá, Maringá, PR,pg403039@uem.br ${ }^{2}$ Engenheiro Agrônomo, Dr. em Ciência do Solo, Universidade Federal da Paraíba, Areia, PB, pereira@ cca.ufpb.br

${ }^{3}$ Engenheiro Agrônomo, Dr. em Ciência do Solo, Embrapa-Solos, Recife, PE, andre.amaral@embrapa.br

${ }^{4}$ Engenheiro Agrônomo, Dr. em Fitotecnia, Universidade Federal da Paraíba, Areia, PB, walterufpb@yahoo.com.br

${ }^{5}$ Bióloga, Dr. em Ciência do Solo, Universidade Federal da Paraíba, Areia, PB, biol.adriana@gmail.com

${ }^{6}$ Eng. Agr., Me. em Ciência do Solo, Universidade Federal Rural de Pernambuco, Recife, PE, danillodutrat@hotmail.com
} 
of conservationist production in the Agreste region of Paraíba state. Soil samples with undisturbed structure were collected in the soil under a system integrating crops, livestock and forests in the layers of 0.00-0.10 and 0.10-0.20 m, in Alagoinha, Paraíba state. The granulometric analysis was carried out with sand fractionation in size classes. Also, the samples were submitted to potentials $-6,-10,-33,-100,-300,-500,-1000$ and -1500 $\mathrm{kPa}$, to determination the water retention curve in the soil and pore distribution by size. The Planosols showed a predominance of the sand fraction in the medium and fine fractions. The pore distribution was heterogeneous according to the sand content. It was concluded that the pores distribution by size presented a positive and significant correlation with the estimated values of the adjustment parameters of the water retention curve in the soil $(n)$ and $(\alpha)$.

Keywords: Porosity, water retention curve, Planosols, ICLF. 


\section{INTRODUÇÃO}

O sistema poroso do solo é caracterizado por uma combinação entre textura e estrutura (Lal e Shukla, 2004). São vazios genéricos formados através da disposição das partículas primárias do solo e de processos secundários relacionados a formação do solo como agregação, atividade da fração argila, congelamento e descongelamento do solo e da atividade biológica (Weninger et al., 2019). Essas estruturas determinam a funcionalidade do solo através de sua atuação na produção de alimentos, armazenamento de água, limpeza de águas residuais e estocagem de carbono no solo (Kravchenko et al., 2019), no comportamento da curva de retenção de água no solo, no coeficiente de permeabilidade (Zhai et al., 2019), na condutividade hidráulica e infiltração de água no solo (Andrade e Stone, 2013).

Alguns métodos têm sido utilizados na determinação do tamanho de poros do solo, a exemplo das imagens computadorizadas e da derivação indireta da curva de retenção de água no solo (Weninger et al., 2019). No método de derivação, a porosidade é determinada através da relação entre a umidade contida no solo $(\theta)$ com um dado potencial matricial aplicado $(\psi)$ (Dexter et al., 2004). Neste método, a distribuição de poros é determinada através dos valores de umidade utilizadas na obtenção da curva de retenção de água no solo, havendo, portanto, uma estreita relação entre esses parâmetros.

Alguns autores correlacionaram o comportamento da curva de retenção de água no solo com a distribuição de poros por tamanho e os parâmetros $(n)$ e $(\alpha)$ da equação matemática proposta por van Genuchten (1980). Horton et al. (1987), por exemplo, enfatizou que o parâmetro (n) relaciona-se com a distribuição de poros por tamanho e que com o aumento de valor desse parâmetro, ocorre redução no tamanho dos poros. Souza et al. (2008) observaram que (n) é um fator de forma dependente da textura do solo. O $(m)$ foi proposto por Mualem (1986) e tem relação com a condutividade hidráulica não saturada do solo.
Em relação ao $(\alpha)$ existem algumas lacunas na literatura a serem sanadas, principalmente no que se refere a real relação desse parâmetro com o funcionamento físico do solo. Autores como Lima et al. (2014) e Mota et al. (2017), afirmaram que o $(\alpha)$ representa o valor inverso à pressão de entrada de ar no poro de maior diâmetro do solo. Em estudo publicado por van Lier et al. (2018), os autores demostraram matemática e graficamente, que o parâmetro $(\alpha)$ é apenas um parâmetro de escala relativo à matriz do eixo potencial, sem relação coma entrada de ar, como frequentemente abordado. Alguns trabalhos disponíveis na literatura relatam que existe uma relação entre a distribuição de poros por tamanho com o comportamento da curva de retenção de água no solo (Silva et al., 2017; Parahyba et al., 2019). Porém, não é conhecido se essa relação pode ocasionar possíveis alterações nos parâmetros de ajustes da curva de retenção proposta por van Genuchten (1980) em Planossolo Háplico com acentuado teor de areia.

Nesse sentido, o objetivo deste trabalho foi avaliar a relação entre a distribuição de poros no solo por tamanho com os parâmetros de ajuste da curva característica de retenção de água no solo $(\alpha, n)$ em um Planossolo Háplico sob manejo de produção conservacionista no Agreste da Paraíba.

\section{MATERIAL E MÉTODOS}

\section{Caracterização da área experimental}

O experimento foi implantado em julho de 2015 em área experimental da Empresa Paraibana de Pesquisa, Extensão Rural e Regularização Fundiária (EMPAER), em Alagoinha, Paraíba, Brasil (06 ${ }^{\circ} 57^{\prime}$ 00" $\mathrm{S}$ e $35^{\circ} 32^{\prime} 42^{\prime} \mathrm{W}$; altitude de 317 metros). Segundo a classificação climática de Köppen-Geiger o clima do município é caracterizado como tropical quente e úmido, tipo As' (Peel et al., 2007), com chuvas de outono-inverno e período de maior precipitação entre os meses de abril e julho (Silva e Nascimento, 2020).

A precipitação média anual é de $995 \mathrm{~mm}$, com temperatura variando de 22 a $26{ }^{\circ} \mathrm{C}$ e umidade 
relativa do ar de $65 \%$. O solo da área experimental foi classificado como Planossolo Háplico Eutrófico mésico solódico com horizonte A moderado e de textura franco-arenosa (Santos et al., 2018). A área experimental encontra-se ocupada com um sistema de integração Lavoura-Pecuária-Floresta (iLPF), contendo os seguintes consórcios em parcelas experimentais com área total de $760 \mathrm{~m}^{2}$ (38 x 20m): I) Brachiaria decumbens + Ipê (Handroanthus impetiginosus) (BI), II) Brachiaria decumbens + Sabiá (Mimosa caesalpiniaefolia) (BS), III) Milho (Zea mays) + Brachiaria decumbens (LA), IV) Brachiaria decumbens + Gliricidia (Gliricidia sepium) (BG) e V) Brachiaria decumbens (BD).

O delineamento experimental utilizado foi o de blocos casualizados (DBC) com cinco tratamentos e quatro repetições, perfazendo um total de 20 parcelas experimentais.

\section{Coleta de mostras e atributos analisados}

As amostras de solo com estrutura indeformada foram coletadas no mês de fevereiro de 2019, logo após o período chuvoso, sob o renque nos tratamentos com arbóreas, com distância de 10 metros entre os pontos amostrais (Figura 1). Utilizou-se cilindros metálicos tipo Uhland com volume de $102,09 \mathrm{~cm}^{3}$, em gride amostral categorizado com quatro pontos em cada parcela, nas profundidades de 0,00-0,10 e 0,10-0,20 m (Figura 1).

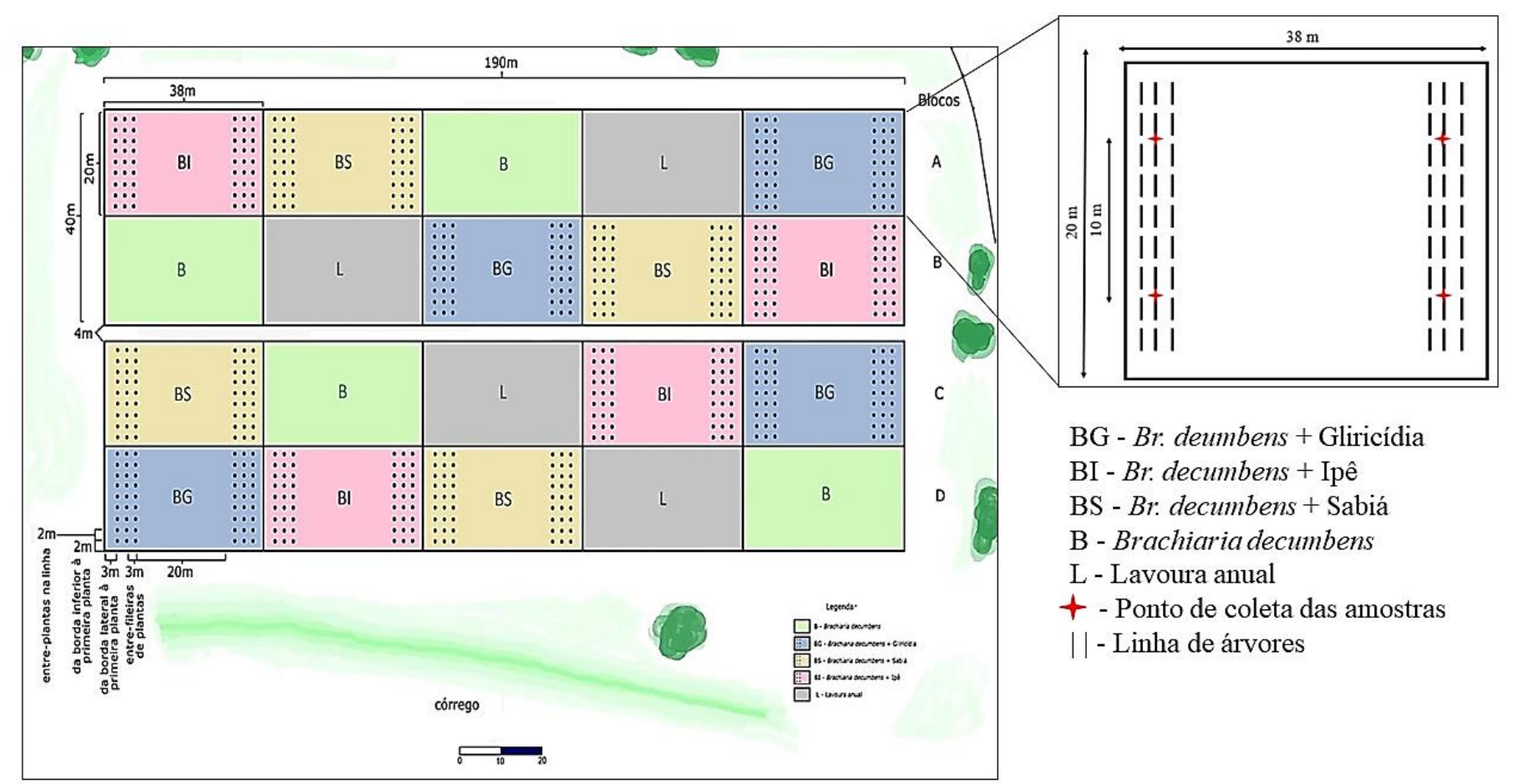

Figura 1. Esquema da área experimental e dos pontos amostrais do Planossolo Háplico, município de Alagoinha (PB).

As amostras de solo foram encaminhadas ao Laboratório de Análises Físicas do Solo do Centro de Ciências Agrárias da Universidade Federal da Paraíba, para a determinação dos seguintes parâmetros: Granulometria (areia fracionada em cinco classes de tamanho: muito grossa, grossa, média, fina e muito fina), silte, argila; curva característica de retenção de água no solo (CRA) e distribuição de poros por tamanho (Ø) e obtido algumas relações: areia grossa/ areia fina (AG/AF), relação silte/ argila (SI/AR). 


\section{Granulometria do solo e CRA}

A granulometria foi determinada em amostras de solo deformadas e secas ao ar (TFSA) usando-se o hidróxido de sódio ( $\left.\mathrm{NaOH} \quad 1 \quad \mathrm{~mol}^{-1}\right)$ como dispersante químico, com posterior agitação mecânica (Teixeira et al., 2017). A areia foi fracionada em cinco classes de tamanho: Areia muito grossa $(2-0,84 \mathrm{~mm})$, grossa $(0,84-0,5 \mathrm{~mm})$, média $(0,5-0,21 \mathrm{~mm})$, fina $(0,21-0,105 \mathrm{~mm})$ e muito fina $(0,105-0,053 \mathrm{~mm})$. Com os dados de granulometria pôde-se calcular a relação areia grossa/ areia fina do solo (AG:AF) e a relação silte/ argila (SI:AR).

As amostras de solo com estrutura indeformada foram saturadas e submetidas aos potenciais: $-6\left(\theta_{6}\right)$; $-10\left(\theta_{10}\right) ;-33\left(\theta_{33}\right) ;-100\left(\theta_{100}\right) ;-300\left(\theta_{300}\right) ;-500$ $\left(\theta_{500}\right) ;-1000\left(\theta_{1000}\right)$ e -1500 $\left(\theta_{1500}\right) \mathrm{kPa}$ em câmara de Richards com placas porosas de forma a umidade volumétrica $(\theta)$ (Klute, 1986). Os dados de umidade foram ajustados utilizando-se o software SWRC (Dourado Neto et al., 2000), empregando-se o modelo matemático proposto por van Genucten (1980):

$$
(\theta)=\theta r+\frac{(\theta s-\theta r)}{\left[\left(1+(\alpha \Psi m)^{n}\right]^{m}\right.}(\text { Equação } 1)
$$

Em que: $\theta$ é o conteúdo volumétrico de água no solo $\left(\mathrm{m}^{3} \mathrm{~m}^{-3}\right)$ correspondente ao potencial matricial aplicado ( $\Psi \mathrm{m}, \mathrm{kPa}) ; \theta$ r representa a umidade residual do solo; $\theta$ s é a umidade na saturação; $\Psi$ é o valor absoluto do potencial matricial $(\mathrm{kPa}) ; \mathrm{n}, \mathrm{m}$ e $\alpha$, são parâmetros empíricos do modelo, com $\alpha$ expresso em $\left(\mathrm{kPa}^{-1}\right) ; \mathrm{n}$ e $\mathrm{m}$ (adimensionais).

A distribuição de poros por tamanho (Ø) foi determinada de acordo com metodologia proposta por Bouma (1991), utilizando a umidade do solo $(\theta)$ correspondentes aos pontos da curva de retenção de água no solo $(\psi)$.

O cálculo da distribuição foi realizado utilizando a seguinte equação:

$$
\mathrm{D}=4 \sigma \operatorname{Cos} \theta / \Psi \mathrm{m}(\text { Equação } 2)
$$

Em que: D é o diâmetro do poro $(\mu \mathrm{m})$; $\sigma$ é a tensão superficial da água $\left(0,272\right.$ bar a $\left.20^{\circ} \mathrm{C}\right) ; \theta$ é o ângulo de contato entre o menisco e a parede do tubo capilar (considerado como zero) e $\Psi_{\mathrm{m}}$ é o potencial matricial aplicado $(\mathrm{kPa})$.

As classes de tamanho dos poros foram subdivididas conforme classificação proposta por Klein e Libardi (2002), sendo $\varnothing>0,05 \mathrm{~mm}(50 \mu \mathrm{m})$ classificado como macroporos, que perdem água em potenciais menores que $-6 \mathrm{kPa}$; $\varnothing$ entre 0,05 e 0,0002 $\mathrm{mm}(50$ a $0,2 \mu \mathrm{m})$ microporos que perdem água entre -6 e $-1500 \mathrm{kPa}$ e, $\varnothing<0,0002 \mathrm{~mm}$, criptoporos, que perdem água em potenciais maiores que $-1500 \mathrm{kPa}$.

\section{Análise estatística}

As médias da análise granulométrica foram avaliadas através da análise estatística descritiva: média, coeficiente de variação $(\mathrm{CV})$ e desvio padrão $(\sigma)$. Realizou-se também, a análise com o teste de Tukey $(\mathrm{p}<0,05)$ utilizando o software $\mathrm{R}$ ( $\mathrm{R}$ Development Core Team, 2013). Na análise de correlação entre as variáveis $(n, \alpha)$ e a distribuição de poros do solo, utilizou-se a técnica de árvore de decisão (códigos R e Python), com o software R.

\section{RESULTADOS E DISCUSSÃO}

Os resultados referentes a granulometria do solo encontram-se apresentados na Tabela 1 . Verifica-se que a areia predominou na camada de 0,00-0,010 m em relação a camada de 0,10-0,20 m, com uma diferença de $14,4 \mathrm{~g} \mathrm{~kg}^{-1}$. Na camada superficial o teor de areia foi da ordem de $688,5 \mathrm{~g} \mathrm{~kg}^{-}$ 1, reduzindo para $674,0 \mathrm{~g} \mathrm{~kg}^{-1}$ camada de $0,10-0,20$ m. Com relação a granulometria da fração areia, verifica-se predomínio de areia fina (AF) em relação a areia grossa (AG) nas duas camadas de solo avaliadas (Tabela 1). Resultado semelhante foi verificado por Parahyba et al. (2019) avaliando a capacidade de retenção de água em Neossolo e Latossolo do semiárido baiano. Eles observaram que esses solos apresentaram maior concentração de areia nas frações média (AME) e fina (AF), que dentre outros benefícios contribui para melhorias na capacidade de armazenamento de água no solo.

Percebe-se que houve aumento na concentração de argila em profundidade, com 148,5 $\mathrm{g} \mathrm{kg}^{-1}$ na 
camada de $0,00-0,10 \mathrm{~m}$ e $180,0 \mathrm{~g} \mathrm{~kg}^{-1}$, na camada de 0,10-0,20 $\mathrm{m}$, diferença de $31,5 \mathrm{~g} \mathrm{~kg}^{-1}$ entre as camadas. Esse é um comportamento característico, pois a concentração de argila nas camadas subsuperficiais de Planossolos é uma característica intrínseca do mesmo, e geralmente ocorre através da perda de argila da camada superficial para a subsuperficial pela eluviação (Santos et al., 2013). Contudo, grande parte dessa argila encontrada nos Panossolos do semiárido é considerada de atividade alta, dificultando o preparo do solo em algumas condições de umidade (Santos et al., 2013).

Tabela 1.Classificação textural e granulometria da fração areia em Planossolo Háplico, município Alagoinha (PB).

\begin{tabular}{|c|c|c|c|c|c|c|c|c|c|}
\hline \multirow{2}{*}{ Tratamento } & AMG & $\mathbf{A G}$ & AME & $\mathbf{A F}$ & AMF & SIL & ARG & AG:AF & SI:AR \\
\hline & \multicolumn{9}{|c|}{ 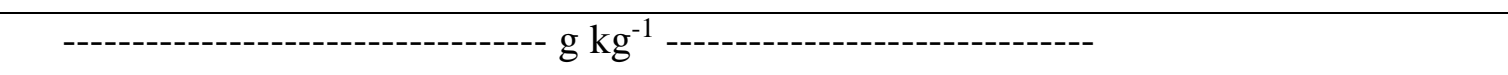 } \\
\hline \multicolumn{10}{|c|}{$0,00-0,10 \mathrm{~m}$} \\
\hline $\mathrm{BI}$ & 38 & 53 & 139 & 317 & 122 & 163 & 143 & 0,157 & 1,13 \\
\hline $\mathrm{BS}$ & 31 & 55 & 148 & 320 & 117 & 162 & 152 & 0,147 & 1,06 \\
\hline LA & 37 & 67 & 171 & 324 & 110 & 162 & 156 & 0,171 & 1,03 \\
\hline BG & 36 & 78 & 180 & 318 & 110 & 167 & 152 & 0,187 & 1,09 \\
\hline $\mathrm{BD}$ & 31 & 55 & 142 & 322 & 117 & 158 & 139 & 0,148 & 1,13 \\
\hline Média & 35 & 62 & 156 & 320 & 115 & 162 & 148 & 0,162 & 1,09 \\
\hline$\sigma$ & 3,10 & 10,71 & 18,37 & 2,86 & 5,16 & 2,38 & 7,09 & 0,017 & 0,04 \\
\hline $\mathrm{CV}(\%)$ & 8,7 & 17,3 & 11,7 & 0,8 & 4,4 & 1,4 & 4,7 & 10,6 & 4,0 \\
\hline \multicolumn{10}{|c|}{$0,10-0,20 \mathrm{~m}$} \\
\hline BI & 32 & 47 & 128 & 303 & 118 & 147 & 177 & 0,143 & 0,83 \\
\hline BS & 33 & 52 & 149 & 317 & 124 & 142 & 166 & 0,144 & 0,85 \\
\hline LA & 49 & 73 & 170 & 308 & 115 & 136 & 169 & 0,205 & 0,80 \\
\hline BG & 32 & 70 & 164 & 303 & 120 & 160 & 190 & 0,173 & 0,84 \\
\hline $\mathrm{BD}$ & 31 & 54 & 136 & 322 & 120 & 144 & 160 & 0,147 & 0,90 \\
\hline Média & 35 & 59 & 149 & 311 & 119 & 146 & 172 & 0,162 & 0,84 \\
\hline$\sigma$ & 7,63 & 11,56 & 17,85 & 8,56 & 3,28 & 8,89 & 11,58 & 0,027 & 0,03 \\
\hline $\mathrm{CV}(\%)$ & 21,5 & 19,5 & 11,9 & 2,7 & 2,7 & 6,1 & 6,7 & 16,5 & 4,1 \\
\hline
\end{tabular}

$\mathrm{AMG}=$ Areia muito grossa, $\mathrm{AG}=$ Areia grossa, $\mathrm{AME}=$ Areia média, $\mathrm{AF}=$ Areia fina, $\mathrm{AMF}=$ Areia muito fina, $\mathrm{SIL}=\mathrm{Silte}, \mathrm{ARG}=\mathrm{Argila}, \mathrm{AG}: \mathrm{AF}$ $=$ Relação areia grossa/ areia fina, SI:AR $\mathrm{r}=$ Relação silte/ argila, BI= Brachiaria decumbens $+\mathrm{Ipê}, \mathrm{BS}=$ Brachiaria decumbens + Sabiá, LA= Lavoura anual + Brachiaria decumbens, $\mathrm{BG}=$ Brachiaria decumbens + gliricídia, $\mathrm{BD}=$ Brachiaria decumbens, $\sigma=\mathrm{Desvio}$ padrão, $\mathrm{CV}=\mathrm{Coeficiente}$ de variação.

Embora baixo, o teor de argila de $148,5 \mathrm{~g} \mathrm{~kg}^{-1}$ a retenção de água no solo. Silva et al. (2019) na camada de 0,00-0,10 m e 180,0 $\mathrm{g} \mathrm{kg}^{-1}$ na camada observaram que em um Planossolo Háplico de textura de 0,10-0,20 m, contribuem consideravelmente para franco-arenosa sob iLPF, o conteúdo de água retido 
foi da ordem de $0,374 \mathrm{~m}^{3} \mathrm{~m}^{-3}$ para a camada de 0,00 $0,10 \mathrm{~m} \mathrm{e} 0,428 \mathrm{~m}^{3} \mathrm{~m}^{-3}$ na camada de $0,10-0,20 \mathrm{~m}$, um incremento de $12,61 \%$ no conteúdo de água armazenado. Esse fato evidencia o quão importante é a granulometria para o funcionamento do solo, principalmente em se tratando do armazenamento e disponibilidade de água nas plantas. A problemática da retenção de água em solos arenosos é muito importante, pois a germinação das sementes e o desenvolvimento das plantas são criticamente restritivos em solos com baixa umidade (Andry et al., 2009).

Foi observado valor médio igual da relação areia grossa/ areia fina para as duas camadas de solo avaliadas, com 0,16 partes de areia grossa (AG) para uma $(1,0)$ parte de areia fina. É uma relação baixa e evidencia o predomínio da areia fina sobre a areia grossa. Para Donagemma et al. (2016), o conhecimento dessa relação (AG:AF) é imprescindível, pois o efeito da ocupação dos espaços vazios entre partículas grandes (AG), por partículas finas (AF), ocasiona o fenômeno conhecido como empacotamento, que leva ao adensamento das camadas de solo e diminui os espaços porosos. Esse efeito pode exercer influência sobre a retenção e disponibilidade de água para as plantas, pois o empacotamento pode levar ao aumento de poros de menor diâmetro.

Para a relação silte/argila (SI:AR), verifica-se que os valores médios foram superiores na camada de 0,00-0,10 m em relação a camada de 0,10-0,20 m, com amplitude de 0,25 entre camadas. Esse resultado vai de acordo com o encontrado por Souza et al. (2010) e Silva et al. (2018) realizando o levantamento da textura do solo e da relação silte/ argila (SI:AR) de solos do semiárido Nordestino. Em ambos os trabalhos a relação encontrada pelos autores foi da ordem de 0,89 a 1,60, sendo maior na camada superficial do solo $(0,00-0,10 \mathrm{~m})$. Para eles, este fato diz respeito ao predomínio da fração areia em relação ao silte e argila na maioria dos solos de clima semiárido, não evidenciando seguramente o grau de intemperismo desses solos. Contudo, é importante ressaltar que a relação silte/argila tem forte ligação com os baixos teores de silte do solo e que valores inferiores a 0,7 em solos de textura arenosa, ou inferior a 0,6 nos solos de textura argilosa a muito argilosa, indicam predomínio avançado do intemperismo (Silva et al., 2018).

Em relação a distribuição de poros por tamanho, verifica-se o predomínio de microporos ( $\varnothing$ 49 a 2,0 $\mu \mathrm{m})$ e criptoporos (Ø $<0,2 \mu \mathrm{m})$ (Tabela 2). Em termos médios, observa-se maior distribuição de poros na camada de $0,00-0,10$ m em relação a camada de 0,10-0,20 m. Verifica-se também uma heterogeneidade na distribuição de poros nesse solo, fato esse influenciado pela concentração de areia média e fina, como destacado por Kiehl (1979). Verifica-se também que houve variação estatística significativa entre as camadas de solo para a distribuição de poros com tamanho de (Ø 49 a $29 \mu \mathrm{m})$ e $(\varnothing<0,2 \mu \mathrm{m})$, sendo superior na camada de 0,00 $0,10 \mathrm{~m}$ (Tabela 2).

Do volume de poros observados na camada de 0,00-0,10 m, 71,23\% é composto por (macroporos e mesoporos), que em termos de potencial seria de 0 a $-100 \mathrm{kPa}$. O restante, $28,77 \%$ foram classificados como poros de menor diâmetro (microporos), onde a água dificilmente será absorvida pela maioria das plantas cultivadas. Na camada de 0,10-0,20 m, observa-se que houve redução nessa relação, sendo $69,75 \%$ composto por poros de maior diâmetro e o restante $30,25 \%$ por poros de menor diâmetro ( $\varnothing$ de $0,9 \mathrm{a}<0,2 \mu \mathrm{m})$. Em termos absolutos, pode-se afirmar que na camada de $0,00-0,10 \mathrm{~m}$ a disponibilidade de água para as plantas foi maior que na camada de 0,10 $0,20 \mathrm{~m}$, provavelmente influenciada pela distribuição de poros ou pela deposição de material orgânico.

Tabela 2.Distribuição de poros por tamanho de um Planossolo Háplico, município de Alagoinha (PB). 


\begin{tabular}{|c|c|c|c|c|c|c|c|c|c|c|}
\hline \multirow{2}{*}{ Trat. } & \multirow{2}{*}{$\mathbf{h}$} & $>49$ & 49 a 29 & 29 a 9 & 9 a 2,9 & 2.9 a 0.9 & 0.9 a 0.6 & 0.6 a 0.3 & 0.3 a 0.2 & $<0.2$ \\
\hline & & Macro & \multicolumn{7}{|c|}{ 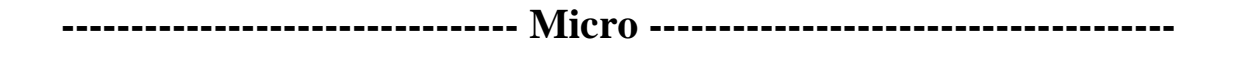 } & Cripto \\
\hline \multicolumn{11}{|c|}{ 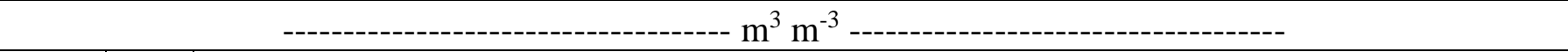 } \\
\hline BI & \multirow{5}{*}{$\begin{array}{l}\Xi \\
0 \\
0 \\
0 \\
0 \\
0 \\
0 \\
0\end{array}$} & $0.069 \mathrm{a}$ & $0.146 \mathrm{a}$ & $0.068 \mathrm{a}$ & $0.020 \mathrm{a}$ & $0.010 \mathrm{a}$ & $0.013 \mathrm{a}$ & $0.006 \mathrm{a}$ & $0.015 \mathrm{a}$ & $0.102 \mathrm{a}$ \\
\hline BS & & $0.073 \mathrm{a}$ & $0.157 \mathrm{a}$ & $0.051 \mathrm{a}$ & $0.020 \mathrm{a}$ & $0.022 \mathrm{a}$ & $0.015 \mathrm{a}$ & $0.002 \mathrm{a}$ & $0.004 \mathrm{a}$ & $0.100 \mathrm{a}$ \\
\hline LA & & $0.081 \mathrm{a}$ & $0.150 \mathrm{a}$ & $0.053 \mathrm{a}$ & $0.023 \mathrm{a}$ & $0.012 \mathrm{a}$ & $0.011 \mathrm{a}$ & $0.002 \mathrm{a}$ & $0.008 \mathrm{a}$ & $0.100 \mathrm{a}$ \\
\hline BG & & $0.073 \mathrm{a}$ & $0.145 \mathrm{a}$ & $0.059 \mathrm{a}$ & $0.027 \mathrm{a}$ & $0.012 \mathrm{a}$ & $0.011 \mathrm{a}$ & $0.001 \mathrm{a}$ & $0.021 \mathrm{a}$ & $0.079 \mathrm{a}$ \\
\hline BD & & $0.077 \mathrm{a}$ & $0.109 \mathrm{a}$ & $0.071 \mathrm{a}$ & $0.018 \mathrm{a}$ & $0.013 \mathrm{a}$ & $0.012 \mathrm{a}$ & $0.007 \mathrm{a}$ & $0.010 \mathrm{a}$ & $0.107 \mathrm{a}$ \\
\hline \multicolumn{2}{|c|}{ Média } & $0,075 \mathrm{a}$ & $0,141 \mathrm{a}$ & $0,060 \mathrm{a}$ & $0,022 \mathrm{a}$ & $0,014 \mathrm{a}$ & $0,012 \mathrm{a}$ & $0,004 \mathrm{a}$ & $0,012 \mathrm{a}$ & $0,098 \mathrm{a}$ \\
\hline \multicolumn{2}{|c|}{$\mathrm{Dp}$} & 0,005 & 0,019 & 0,009 & 0,004 & 0,005 & 0,002 & 0,003 & 0,007 & 0,011 \\
\hline \multicolumn{2}{|c|}{ CV (\%) } & 6,1 & 13,2 & 14,6 & 16,2 & 34,1 & 13,4 & 75,0 & 56,7 & 11,0 \\
\hline & & $>49$ & 49 a 29 & 29 a 9 & 9 a 2,9 & 2.9 a 0.9 & 0.9 a 0.6 & 9 a 0.3 & 0.3 a 0.2 & $<0.2$ \\
\hline & & Macro & & & & Micr & & & & Cripto \\
\hline BI & \multirow{5}{*}{ 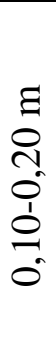 } & $0.066 a$ & $0.089 a$ & $0.054 a$ & $0.036 \mathrm{a}$ & $0.019 a$ & $0.007 \mathrm{a}$ & $0.009 \mathrm{a}$ & $0.011 \mathrm{a}$ & $0.098 \mathrm{a}$ \\
\hline BS & & $0.058 \mathrm{a}$ & $0.142 \mathrm{a}$ & $0.047 \mathrm{a}$ & $0.036 \mathrm{a}$ & $0.012 \mathrm{a}$ & $0.009 \mathrm{a}$ & $0.005 \mathrm{a}$ & $0.009 \mathrm{a}$ & $0.090 \mathrm{a}$ \\
\hline LA & & $0.068 \mathrm{a}$ & $0.116 a$ & $0.051 \mathrm{a}$ & $0.026 \mathrm{a}$ & $0.015 \mathrm{a}$ & $0.010 \mathrm{a}$ & $0.002 \mathrm{a}$ & $0.008 \mathrm{a}$ & $0.103 \mathrm{a}$ \\
\hline BG & & $0.052 \mathrm{a}$ & $0.153 \mathrm{a}$ & $0.072 \mathrm{a}$ & $0.019 \mathrm{a}$ & $0.011 \mathrm{a}$ & $0.010 \mathrm{a}$ & $0.002 \mathrm{a}$ & $0.023 \mathrm{a}$ & $0.077 \mathrm{a}$ \\
\hline BD & & $0.064 a$ & $0.094 \mathrm{a}$ & $0.071 \mathrm{a}$ & $0.020 \mathrm{a}$ & $0.015 \mathrm{a}$ & $0.013 \mathrm{a}$ & $0.003 \mathrm{a}$ & $0.023 \mathrm{a}$ & $0.105 \mathrm{a}$ \\
\hline \multicolumn{2}{|c|}{ Média } & $0,061 \mathrm{a}$ & $0,118 b$ & 0,059 a & $0,027 \mathrm{a}$ & $0,014 \mathrm{a}$ & 0,009 a & $0,004 \mathrm{a}$ & $0,014 \mathrm{a}$ & $0,094 \mathrm{~b}$ \\
\hline \multicolumn{2}{|c|}{$\mathrm{Dp}$} & 0,006 & 0,028 & 0,011 & 0,008 & 0,003 & 0,002 & 0,002 & 0,007 & 0,011 \\
\hline \multicolumn{2}{|c|}{$\mathrm{CV}(\%)$} & 10,6 & 23,8 & 19,8 & 30,2 & 21,7 & 22,1 & 70,2 & 51,1 & 12,0 \\
\hline
\end{tabular}

$\mathrm{BI}=$ Brachiaria decumbens + Ipê, $\mathrm{BS}=$ Brachiaria decumbens + Sabiá, $\mathrm{LA}=$ Lavoura anual, $\mathrm{BG}=$ Brachiaria decumbens $+\mathrm{gliricídia,} \mathrm{BD}=$ Brachiaria decumbens.

Com relação as camadas, verifica-se que a redução dos poros de maior diâmetro foi de 10,58\% com o aumento de profundidade. Esse fato pode estar relacionado com o aumento de densidade do solo em subsuperfície, como observado por Silva et al. (2019), avaliando a qualidade física do solo nesta área de estudo.

Verifica-se na Tabela 2 que os microporos, classe correspondente à faixa de diâmetro $(\varnothing 0,9$ a 0,2 $\mu \mathrm{m})$ foi de $0,028 \mathrm{~m}^{3} \mathrm{~m}^{-3}$ na camada de $0,00-0,10$ e de $0,027 \mathrm{~m}^{3} \mathrm{~m}^{-3}$ na camada de $0,10-0,20 \mathrm{~m}$, portanto, matematicamente iguais (Tabela 2). A distribuição heterogênea em potenciais menos negativos $\mathrm{e}$ homogênea em potenciais mais negativos demonstra que a distribuição de poros exerce influência sobre o comportamento das curvas de retenção de água no solo (Figura 2). A distribuição de poros na ordem de $0,312 \mathrm{~m}^{3} \mathrm{~m}^{-3}$ na camada de $0,00-0,10 \mathrm{~m}$ e $0,279 \mathrm{~m}^{3}$ $\mathrm{m}^{-3}$ na camada de $0,10-0,20 \mathrm{~m}$, condicionaram o comportamento da CRA próximo a saturação, evidenciando a influência da distribuição dos poros por tamanho sobre esta variável. Resultados semelhantes foram encontrados por Ribeiro et al. (2007) e Parahyba et al. (2019). 


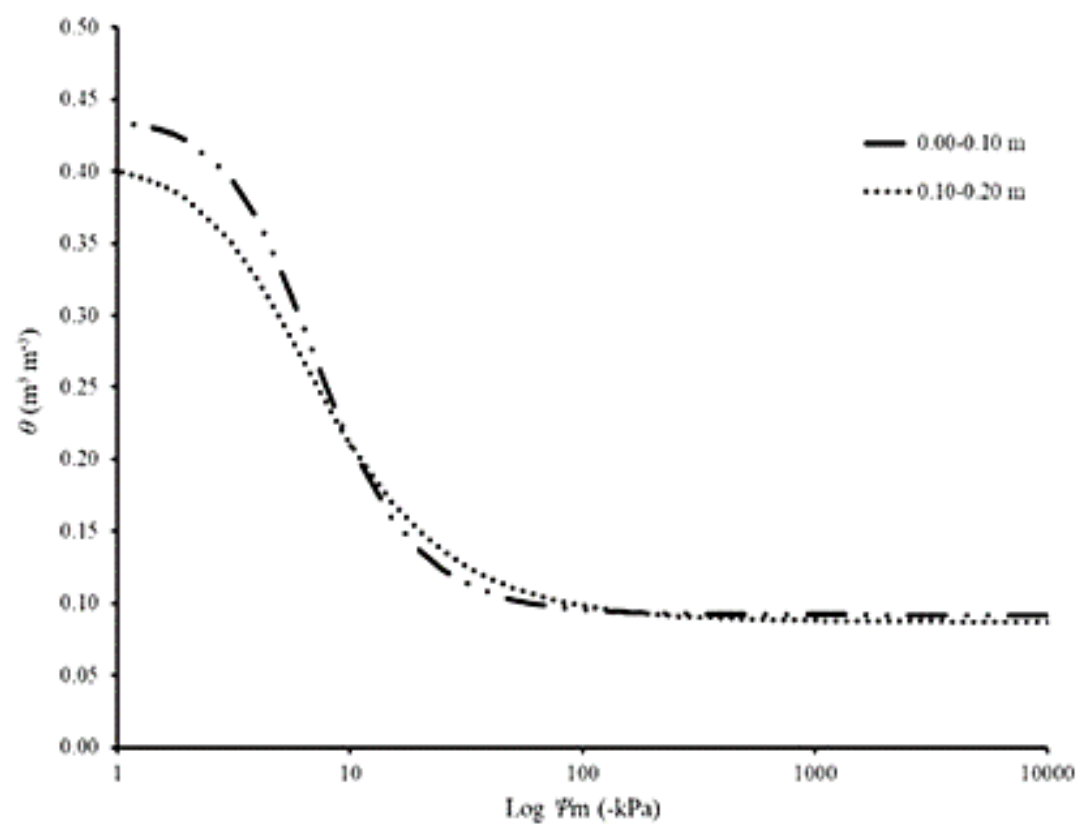

Figura 2. Curva de retenção de água no solo (CRA) de um Planossolo Háplico, município de Alagoinha (PB).

A respeito do efeito das espécies florestais sobre o comportamento das curvas de retenção de água no solo e na distribuição de poros por tamanhos, pode-se afirmar, baseado em resultados apresentados por Silva et al. (2019), que a maior concentração de carbono orgânico em superfície contribuiu para a melhoria desses atributos. É evidente que teores consideráveis de COT melhoram a estrutura do solo, a distribuição de poros em classes de tamanho bem definidas, além de contribuir para melhoria no armazenamento e disponibilidade de água às plantas.

Em relação aos criptoporos $(\varnothing<0,2 \mu \mathrm{m})$, verifica-se que houve diferença estatística significativa entre as camadas de solo, sendo superior na camada de $0,00-0,10 \mathrm{~m}$. Os critoporos são uma classe de poros de vital importância para o solo; contudo, Klein (1998) afirma que são poros nos quais a água permanece retida com energia muito alta, sendo, portanto, dispensável para as plantas. Nessa faixa, a água está retida em um potencial abaixo do ponto de murcha permanente $(<-1500 \mathrm{kPa})$, portanto, indisponível para a maioria das plantas cultivadas (Klein, 1998).

Em relação aos parâmetros de ajustes da curva de retenção de água no solo $(n, \alpha)$ e a distribuição de poros no solo por diâmetro, verifica-se na Figura 3 que houve correlação positiva entre esses parâmetros, com valores superiores a 0,800. Esse resultado corrobora com Horton (1987) e Souza et al. (2018) ao afirmarem que a distribuição de poros por tamanho influencia no comportamento da CRA e nos parâmetros de ajuste. A correlação apresentada pelos valores estimados, demonstra, claramente, o efeito da distribuição dos poros por tamanho nos valores dos parâmetros de ajustes. Verifica-se na Figura 3 que houve uma correlação linear bem definida entre os parâmetros $(n, \alpha)$ e os poros com tamanho $(\varnothing<0,2$ $\mu \mathrm{m})$. Observa-se que os pontos (valores medidos) se ajustaram perfeitamente sobre a reta. Esse comportamento deve estar relacionado à baixa variabilidade de microporos e criptoporos no ambiente, pois sua presença no solo é função da textura do solo e não do manejo adotado.

Correlações positivas evidenciam que mudanças na melhoria da distribuição dos poros influencia também nos valores médios desses parâmetros. Portanto, pode-se afirmar que práticas de manejo que favoreçam melhorias na distribuição de poros por tamanho são fundamentais para o bom funcionamento físico-hídrico do solo. A correlação positiva entre esses parâmetros pode servir de suporte 
para entender o comportamento das curvas de retenção de água no solo.
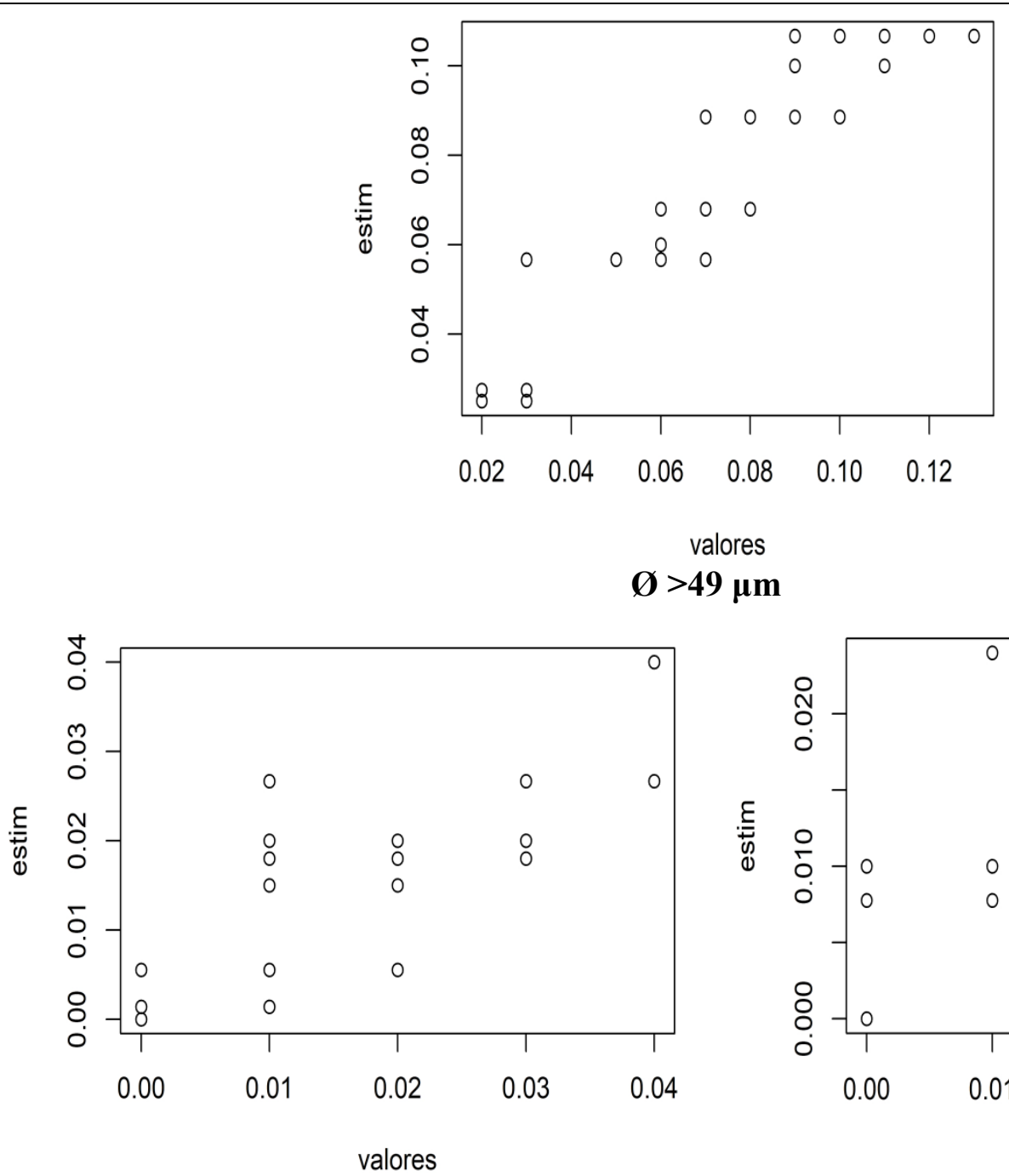

Ø 49-29 $\mu \mathrm{m}$
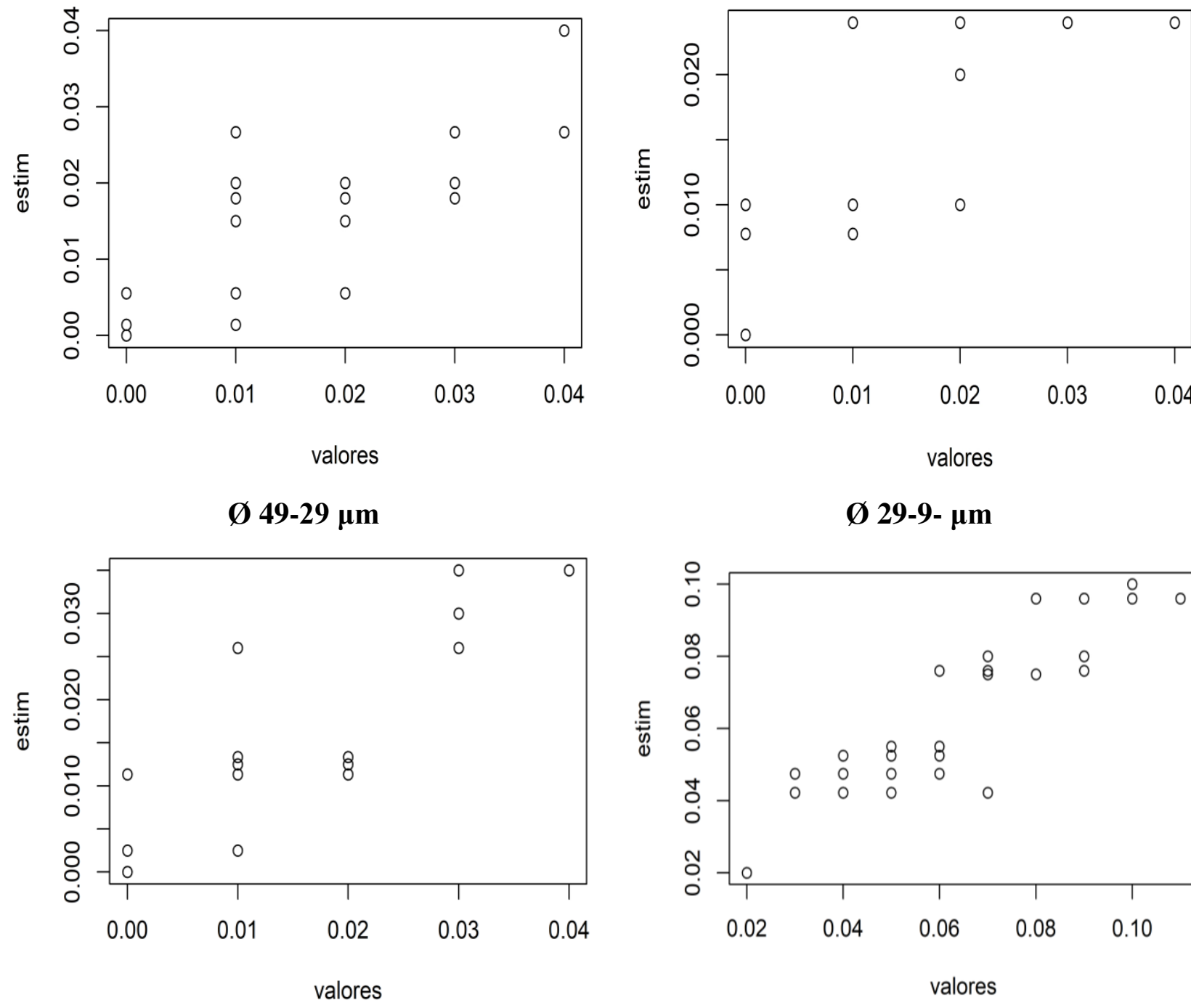

Ø 9-2,9 $\mu \mathrm{m}$

Ø 29-9- $\mu \mathrm{m}$

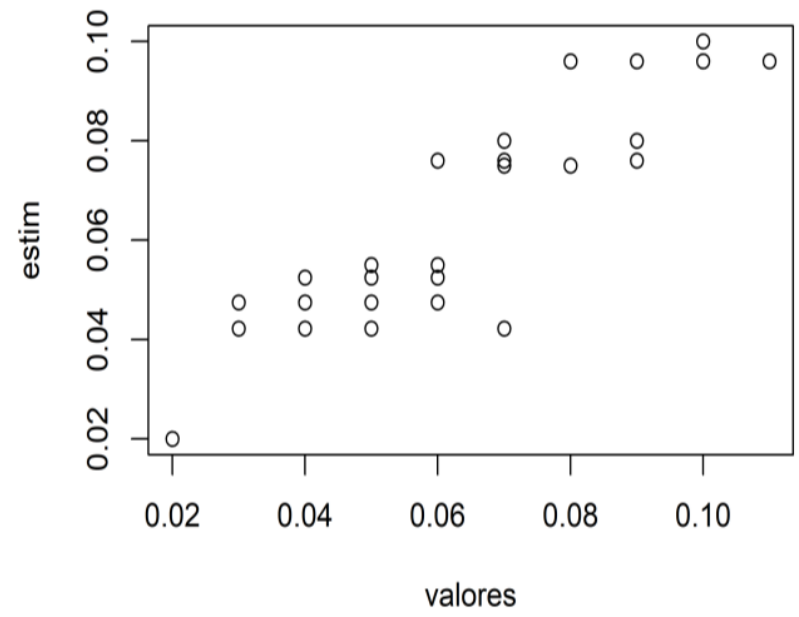

Ø 2,9-0,9 $\mu \mathrm{m}$ 


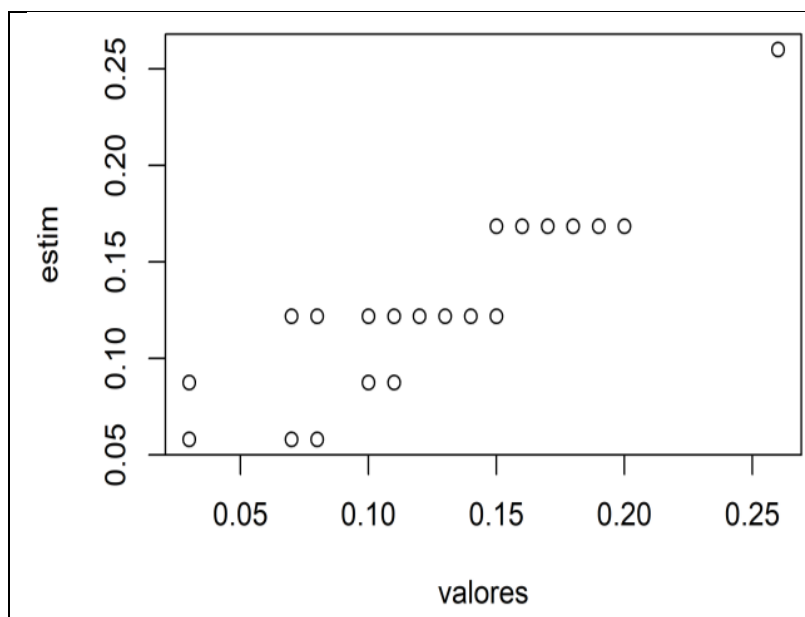

$\emptyset$ 0,9-0,6 $\mu \mathrm{m}$

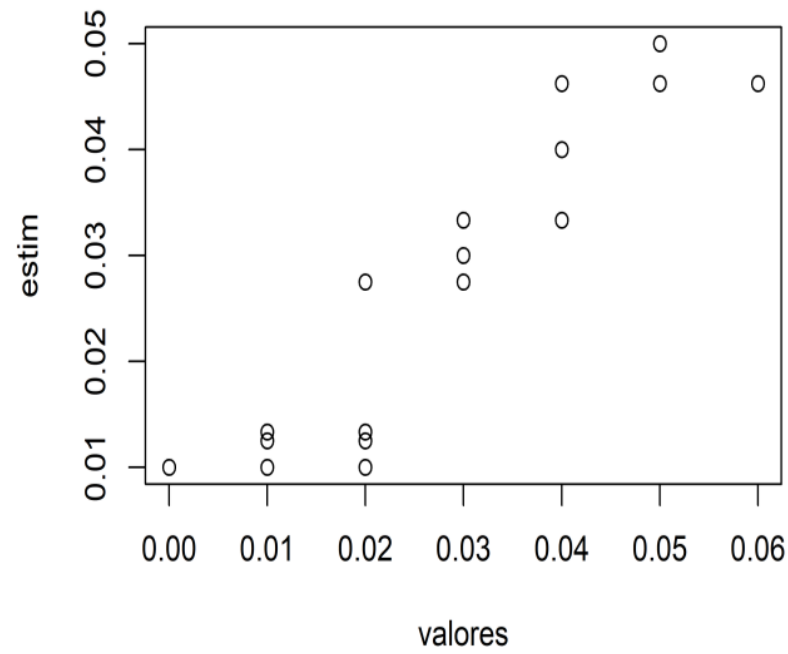

Ø 0,3-0,2 $\mu \mathrm{m}$

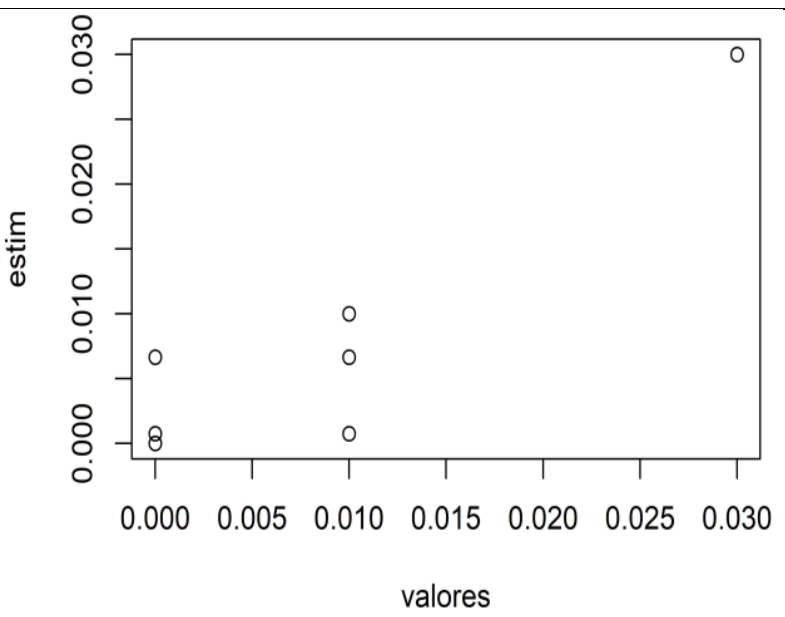

Ø 0,6-0,3 $\mu \mathrm{m}$

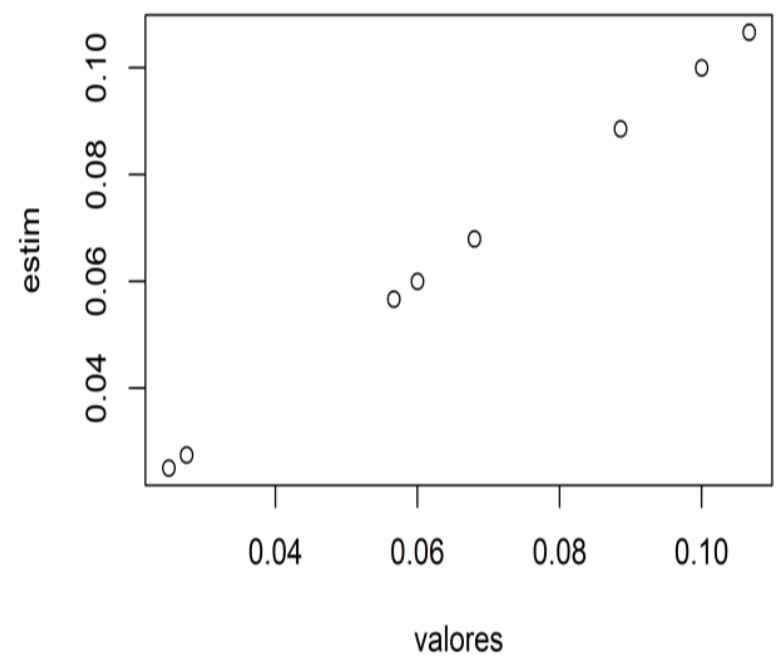

$\emptyset<0,2 \mu \mathrm{m}$

Figura 3. Correlação entre valores estimados de $(n, \alpha)$ com a distribuição de poros no solo por tamanho $(\varnothing)$ de um Planossolo Háplico, município de Alagoinha (PB).

\section{CONCLUSÕES}

Conclui-se que o Planossolo Háplico apresentou classificação textural franco-arenosa com predomínio da areia média e fina. $\mathrm{O}$ predomínio da areia fina exerceu influência sobre a relação areia grossa/areia fina e relação silte/argila do solo, demonstrando haver baixo grau de intemperismo e distribuição heterogênea dos poros por tamanho.

Em superfície, $71,23 \%$ dos poros foram classificados como macroporos e mesoporos e em subsuperfície esse valor reduziu para $69,75 \%$. A distribuição de poros por tamanho tornou-se heterogênea em potenciais menos negativos, e homogênea nos mais negativos.

Houve correlação positiva entre a distribuição de poros por tamanho com os parâmetros de ajustes da curva de retenção de água no solo. A distribuição de poros apresenta estreita relação com os parâmetros n e $\alpha$ da equação de ajuste da curva de retenção para o Planossolo Háplico com elevada concentração de areia.

\section{REFERÊNCIAS BIBLIOGRÁFICAS}


Andrade, R.S.; Stone, L.F. \& Godoy, S.G. (2013). Estimativa da resistência do solo à penetração baseada no índice $\mathrm{S}$ e no estresse efetivo. Revista Brasileira de Engenharia Agrícola e Ambiental. 17(9): 932-937.

Andry, H.; Yamamoto, T.; Irie, T.; Moritani, S.; Inoue, M.; Fujiyama, H. (2009). Water retention, hydraulic conductivity of hydrophilic polymers in sandy soil as affected by temperature and water quality. Journal of Hydrology.373(1): 177-183

Bouma, J. (1991). Influence of soil macroporosity on environmental quality. Advances in Agronomy. 46(1): 1-37.

Dexter, A.R. (2004). Soil physical quality part I. Theory, effects, density and organic matter and effects on root growth. Geoderma. 120(3-4): 201214.

Donagemma, G.K. et al. (2016). Caracterização, potencial agrícola e perspectivas de manejo de solos leves no Brasil. Pesquisa Agropecuária Brasileira. 51(9): 1003-1020.

Dourado-Neto, D.; Nielsen, D.R.; Hopmans, J.W.; Reichardt, K. \& Bacchi, O.O.S. (2000). Software to model soil water retention curve (SWRC version 3.00). Scientia Agricola, 57(1): 191-192.

Horton, R.; Thompson, M.L. \& Mcbride, J.F. (1987). Method of estimating the travel time of no interacting solutes through compacted soil material. Soil Science Society of America Journal. 51(1): 48-53.

Kiehl, E.J. (1979). Manual de Edafologia: relação solo-planta. Piracicaba: Ceres. 262p.

Klein, V.A. \& Libardi, P.L. (2002). Densidade e distribuição do diâmetro dos poros de um Latossolo Vermelho sob diferentes sistemas de uso e manejo. Revista Brasileira de Ciência do Solo. 26(1): 857867.

Klein, V.A. (1998). Propriedades físico-hídricomecânicas de um Latossolo Roxo, sob diferentes sistemas de uso e manejo (Tese de doutorado). Escola Superior de Agricultura de Luiz de Queiroz. Piracicaba, SP, Brasil. p. 162.
Klute, A. (1986). Water retention: laboratory methods. In: Black, C.A. (eds.). Methods of Soil Analysis: I. Physical and Mineralogical Methods. Vol. I. Madison: American Society of Agronomy, Soil Science Society America Journal. p. 635-662.

Kravchenko, A.N., Guber, A.K., Razavi, B.S.; Koestel, J.; Quigley, M.Y.; Robertson, G.P. \& Kuzyakov, Y. (2019). Microbial spatial footprint as a driver of soil carbon stabilization. Nature Communications. 10(3121): 1-10.

Lal, R. \& Shukla, M.K. (2004). Principles of Soil Physics. New York Marcel Dekker.736p.

Lima, J.R.S.; Souza, E.S.; Antonino, A.C.D.; Silva, I.F.; Corrêa, M.M.; Lira, C.A.B. (2014). Atributos físico-hídricos de um Latossolo Amarelo cultivado e sob mata nativa no Brejo Paraibano. Revista Brasileira de Ciências Agrárias. 9(2): 599-605.

Mota, J.C.A.; Libardi, P.L.; Brito, A.S.; Moraes, S.O.; Nascimento, I.V. \& Alencar, T.L. (2017). Variabilidade espacial dos parâmetros da equação de van Genuchten em um Latossolo Vermelho Amarelo.

Agro@mbiente On-line. 11(2): 92-100.

Mualem, Y. (1986). Hydraulic conductivity of unsaturated soils: prediction and formulas. In: KLUTE, A. (Ed.). Methods of soil analysis: I. Physical and mineralogical methods. Madison: American Society of Agronomy - Soil Science American Journal. p.799-823.

Parahyba, R.B.V.; Araújo, M.S.B.; Almeida, B.G.; RolimNeto, F.C.; Sampaio, E.V.S.B. \& Caldas, A.M. (2019). Water retention capacity in Arenosols and Ferralsols in a semiarid area in the state of Bahia, Brazil. Anais da Academia Brasileira de Ciências. 91(4): 1-20.

Peel, M.C.; Finlayson, B.L. \& McMahon, T.A. (2007). Updated world map of the Köppen-Geiger climate classification. Hydrology and Earth System Sciences. 11(4): 1633-1644.

R. Development Core Team. (2013). A language and environment for statistical computing. $R$ Foundation for Statistical Computing, Vienna AUT. Available at: https://www.r-project.org/. 
Ribeiro, K.D.; Menezes, S.M.; Mesquita, M.G.B.F. \& Sampaio, F.M.T. (2007). Propriedades físicas do solo, influenciadas pela distribuição de poros, de duas classes de solo da região de Lavras-MG. Ciência \& Agrotecnologia. 31(11): 1167-1175.

Santos, H.G.; Jacomine, P.K.T.; Anjos, L.H.C.; Oliveira, V.Á.; Lumbreras, J.F.; Coelho, M.R.; Almeida, J.A.; Araújo Filho, J.C.; Oliveira, J.B. \& Cunha, T.J.F. (2018). Sistema Brasileiro de Classificação de Solos. 5. ed. Brasília: Embrapa, 356p.

Silva, É.A.; Benevenute, P.A.N. \& Melo, L.B.B. (2017). Qualidade física de um Latossolo estimada por estudos de curvas de retenção de água, indicadores de fácil determinação e índice $S$. Tecnologia \& Ciência Agropecuária. 11(4): 49-54. Silva, P.L.F.; Oliveira, F.P.; Tavares, D.D.; Nóbrega, C.C. \& Amaral, A.J. (2019). Water availability in a Planosol under integrated crop-livestock-forestry system in the Agreste region of Paraíba, Brazil. Revista Caatinga. 32(2): 449-457.

Silva, P.L.F.; Nascimento, R.S. (2020). Balanço hídrico climatológico e índices de aridez para municípios da microrregião de Guarabira, Paraíba. Brazilian Journal of Biosystems Engineering. 14(2): 125-139.

Silva, W.T.M.; Alves, F.J.B.; Leonardo, F.A.P.; Santos, R.V. \& Farias Júnior, J.A. (2018). Levantamento da textura do solo e da relação silte/argila em regiões semiáridas do Nordeste. Agropecuária Científica do Semiárido. 14(4): 266272.

Souza, A.R.; Albuquerque, S.F.; Lopes, G.M.B.; Silva, A.B. \& Nunes Filho, J. (2013). Caracterização e interpretação de um Planossolo Háplico eutrófico do Agreste pernambucano. Anais da Academia Pernambucana de Ciência Agronômica. 10(1): 271-279.

Souza, E.S.; Antonino, A.C.D.; Ângulo-Jaramillo, R.; Maciel Netto, A.; Montenegro, S.M.G.L. \& Silva, E.B. (2008). Variabilidade espacial dos parâmetros hidrodinâmicos de duas parcelas agrícolas no estado da Paraíba. Revista Brasileira Ciência do Solo. 32(1): 1795-804.
Souza, R.V.C.C.; Ribeiro, M.R.; Souza Junior, V.S.; Corrêa, M.M.; Almeida, M.C.; Campos, M.C. C.; Ribeiro Filho, M.R. \& Schulze, M.B.B. (2010). Caracterização de solos em uma topoclimossequência no maciço de Triunfo - Sertão pernambucano. Revista Brasileira de Ciência do Solo. 34(1): 12591270.

Teixeira, P.C.; Donagemma, G.K.; Fontana, A. \& Teixeira, W.G. (2017). Manual de métodos e análise de solos. Brasília: Embrapa, 573p.

van Genuchten, M.T. (1980). A closed- form equation for predicting the hydraulic conductivity on unsaturated soil. Soil Science Society of American Journal. 44(3): 892-897.

Van Lier, Q.J. \& Pinheiro, E.A.R. (2018). Na alert regarding common misinterpretation of the van Genuchten $\alpha$ parameter. Revista Brasileira de Ciência do Solo. 42(1): 1-5.

Weninger T.; Kreiselmeier, J.; Chandrasekhar, P.; Julich, S.; Feger, K.H.; Schwärzel, K.; Bodner, G. \& Schwen, A. (2019). Effects of tillage intensity on pore system and physical quality of silt-textured soils detected by multiple methods. Soil Research. 57(1): 703-711.

Zhai, G.; Shirzaei, M.; Manga, M. \& Chen, X. (2019). Pore-pressure diffusion, enhanced by poroelastic stresses, controls induced seismicity in Oklahoma. Proceedings of the National Academy of Sciences. 116(33): 16228-16233. 